\title{
A Joint Network-Channel Coding Technique for Single-Hop Wireless Networks
}

\author{
Tuan Tran, Thinh Nguyen and Bella Bose \\ School of EECS, Oregon State University \\ Corvallis, OR 97331, USA \\ \{trantu, thinhq\}@eecs.oregonstate.edu; bose@cs.orst.edu
}

\begin{abstract}
Reliable transmission over an error-prone channel is typically accomplished via channel coding or retransmission of the lost information. In this paper, we investigate a joint network-channel coding technique to increase the bandwidth efficiency of wireless networks. In particular, we show that the proposed joint network-channel coding approach which combines the recent Network Coding (NC) concept with the traditional Forward Error Correction (FEC) technique, can increase the bandwidth efficiency in single-hop wireless networks such as WLAN or WiMAX networks. We present some analytical results on the bandwidth efficiency for both broadcast and unicast scenarios. Based on these theoretical results, we provide a heuristic algorithm that dynamically selects the optimal level of FEC to be used with network coding technique for given channel conditions. For typical channel characteristics, both simulations and theoretical results confirm that the proposed joint networkchannel coding approach can reduce the bandwidth usage up to five times over the Automatic Repeat reQuest (ARQ) technique and up to two times over the HARQ technique.
\end{abstract}

\section{INTRODUCTION}

Traditional approaches to transmit information reliably and effectively over an error-prone network employ either Auto Repeat reQuest (ARQ), Forward Error Correction (FEC), or Hybriad ARQ (HARQ) techniques [1]. Using retransmission approach, the source simply rebroadcasts the lost data if there is at least one receiver not receiving the correct data. This approach assumes that the receivers can somehow communicate to the source whether or not it receives the correct data. On the other hand, using the FEC approach, the source encodes additional information together with the data before broadcast them to the receivers. If the amount of lost data is sufficiently small, a receiver can recover the lost data using some decoding schemes. A HARQ approach combines both of those techniques.

Recently, the Network Coding (NC) approaches to wireless mesh networks, [2][3][4][5] have demonstrated a significant bandwidth improvement over the traditional schemes. The key idea to improve bandwidth efficiency for wireless mesh networks using network coding consists of (a) allowing every node to listen and cache data being transmitted to its neighbor nodes and (b) using the cached information of its neighbors, a node is to broadcast the appropriate coded packets such that with one transmission, many of its neighbors can recover their intended data.

Based on this approach, in [6], we proposed some network coding techniques to increase the bandwidth efficiency of a broadcast session in a single-hop wireless network such as Wireless Local Area Networks (WLAN). In this approach, the AP (Access Point) maintains a queue of lost packets, and combine different lost packets from different receivers in such a way to allow multiple receivers to recover their lost packets simultaneously with one transmission from the AP. In this paper, we extend and improve our previous results with a joint optimization of channel coding and network coding. Our contributions include (a) some analytical results on the bandwidth efficiency for both broadcast and unicast scenarios and (b) a heuristic algorithm that dynamically selects the optimal combination of FEC and $\mathrm{NC}$ for the given channel conditions. In particular, our paper addresses the following question: Given the channel characteristics, how should one maximize the useful bandwidth of a single-hop wireless network? For typical channel characteristics, both simulations the theoretical results confirm that the proposed joint networkchannel coding approach can reduce the bandwidth usage up to five times over the Automatic Repeat reQuest (ARQ) technique and up to two times over the HARQ technique.

The organization of our paper is as follows. We first discuss a few related work in Section II. In Section III, we describe the problem formulation in the context of WLAN/WiMAX networks. In Section IV, we provide some theoretical analysis on the performance of ARQ, HARQ, NC, and network-channel (NC-HARQ) techniques under different channel conditions. Based on these analysis, we describe a heuristic algorithm that dynamically chooses the optimal amount of redundancy to be used with NC in Section V. Simulation results and discussions are provided in Section VI. Finally, we conclude with few remark in Section VII.

\section{RELATED WORK}

This paper is a follow-up work of [6]. In [6], we proposed a network coding scheme to increase the bandwidth efficiency of a wireless broadcast session. In this paper, we investigate a joint network-channel coding technique for both wireless broadcast and unicast sessions. Our work is rooted in the recent development of network coding for wireless ad hoc networks [2][7][8][5]. In [2], Wu et al. proposed the basic scheme that uses XOR of packets to increase the bandwidth efficiency of a wireless mesh network. In [7], Katti et al. implemented an XOR-based scheme in a wireless mesh network and showed a substantial bandwidth improvement over the current approach.

Our work is also related to the wireless broadcast model proposed by Eryilmaz et al. [9]. In this work, Eryilmaz et al. proposed a random network coding scheme for multiple users downloading a single file or multiple files from a wireless base station. Rather than using XOR operations, their scheme encodes every packet using coefficients taken randomly from a 


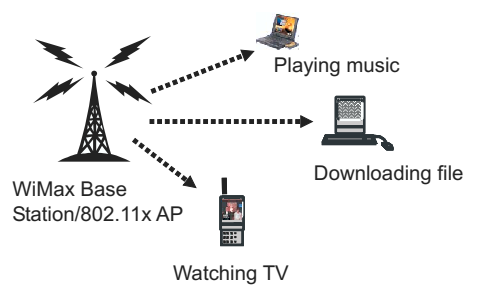

Fig. 1. An example of single-hop network.

sufficiently large finite field [10][11]. This scheme guarantees that the receivers can decode the original data with high probability. Another work is somewhat related to ours is that of Ghaderi et al.[12]. In [12], the authors analyzed the reliability benefit of network coding for reliable multicast by computing the expected number of transmissions using link-by-link ARQ compared to network coding.

In addition, there are other works on multi-hop wireless network with multiple unicast sessions, Li et al. [13][14] have proved that network coding can provide marginal benefits over the approaches that do not use network coding. Also, Lun et al. [15] shows a capacity-approaching coding scheme for unicast or multicast over lossy packet networks in which all nodes perform opportunistic coding by constructing encoded packets with random linear combinations of previously received packets. There is also a rich literature on ARQ, FEC, and HARQ schemes for wireless networks [16][17][18].

\section{PROBLEM DESCRIPTION}

We first begin with a set of assumptions on channel model and protocols.

\section{A. Assumptions}

1) There are one source and $R>1$ receivers, e.g., an AP and number of wireless devices in a WLAN as shown in Figure 1.

2) Data is assumed to be sent in packets, and each packet is sent in a time slot of fixed duration.

3) The source assumes to know which packet from which receiver is lost. This can be accomplished through the use of positive and negative acknowledgments (ACK/NAKs). For simplicity, we assume all the ACK/NAKs are instantaneous, i.e., the source knows (a) whether or not a packet is lost and (b) identity of the receiver with the lost packet instantaneously. This implicitly assumes that ACK/NAKs are never lost. This assumption is not critical as we can easily incorporate the delay and bandwidth used by ACK/NAKs into the analysis. In addition, we assume that CRC with sufficiently large width $r$ (bits) is used for every packet, such as the probability of an undetectable bit error within a packet is virtually zero.

4) We assume that the packet loss at a receiver $i$ follows the Bernoulli distribution with parameter $p_{i}$. Furthermore, the packet losses at these receivers are uncorrelated. This model is clearly insufficient to describe many real-world scenarios. One can develop a more accurate model, albeit complicate analysis.
For comparison purposes, we investigate the performance of ARQ, HARQ, Network Coding (NC), Network-Channel (NCHARQ) for two scenarios: broadcast and unicast.

Broadcast Scenario. The source has set of $M$ distinct packets and each receiver wants all $M$ packets.

Unicast Scenario. The source has a set of $M \times R$ distinct packets, and each receiver wants a disjoint subset consisting of $M$ packets.

Under these settings, we want to characterize the time required for each technique to successfully deliver all the intended packets to all the receivers for given channel characteristics. We assume a fixed underlying physical bandwidth, and therefore the time required to successfully transmit all the packets to the intended receivers can be characterized by ratio of the number of data bits to the actual transmitted bits. Based on this, all schemes under investigation will use the following definition of the bandwidth efficiency as the evaluating metric.

Definition 3.1: The bandwidth efficiency is defined as the ratio of the number of successfully transmitted data bits to that of the actual transmitted bits.

By definition, the number of actual transmitted bits is always greater than or equal to the number of data bits due to the addition of either retransmitted bits or parity bits introduced by FEC. Thus, a scheme $A$ is better than scheme $B$ if it results in higher bandwidth efficiency. Furthermore, no scheme can have a bandwidth efficiency that is greater than 1 .

\section{AnAlysis of Transmission TEChNiQues}

In this section, we provide some theoretical analysis for the ARQ, HARQ, NC, and NC-HARQ techniques for both broadcast and unicast scenarios. For the sake of expository simplicity, we present the analysis for the case of one sender and two receivers. An analysis for the general case of $R>2$ receivers can be found in [19].

We emphasize that there is a number of parameters associated with each technique. The values of these parameters affect the bandwidth efficiency of a particular technique. For example, the bandwidth efficiency of the retransmission technique is greatly influenced by the packet size being used, while the performance of the HARQ technique depends on the amount of redundancy used. Although one can find the optimal parameters to obtain the highest bandwidth efficiency for each technique under the given network conditions, and use these parameters for comparison among different techniques, doing so may not be practical in other aspects. For example, the optimal packet size to achieve the highest bandwidth efficiency for ARQ technique might be too small or too large to be efficiently realized in hardware. Therefore, the aim of this section is to provide the analytical expressions for the bandwidth efficiencies of different transmission techniques as a function of their parameters, and omit the optimal selections of these parameters. When comparing the performance of two techniques, we will provide the justification for choosing the ranges of the parameters that make the most sense.

To aid the analysis, we define the following terms:

- $p_{i}$ : The bit error rate at receiver $R_{i}$ (recall that we assume the bit error event has a Bernoulli distribution.)

- $P_{i}$ : The packet error rate at receiver $R_{i}$ when FEC is not employed. $P_{i}$ is a function of $p_{i}$ and the packet size. 
- $P_{i}^{f}$ : The packet error rate at receiver $R_{i}$ when FEC is employed. It is a function of $p_{i}$, the packet size, and the FEC protection level.

- $N$ : Packet size in bits, including all parity bits. $N$ is assumed to be the same for all techniques and receivers.

- $L_{i}$ : The number of data bits in a packet intended for receiver $R_{i}$.

- $R S(n, k)$ : Reed-Solomon code with $k$ data symbols and $n-k$ redundant symbols.

- $m$ : The number of bits per FEC symbols.

- $r$ : The number of bits in CRC used to detect bit error in every packet. Every scheme uses the same number of CRC bits.

\section{A. Automatic Repeat reQuest (ARQ)}

Using the ARQ scheme, the sender sends packets in sequence. If a packet loss occurs at some receiver, the receiver will send a NAK message to the sender to signal the sender to rebroadcast that lost packet. Our goal is to compute the bandwidth efficiency of this scheme, given the bit error rates at different receivers and the packet size. We assume that a packet loss occurs when there is at least one bit error within a packet. Thus, the packet error probability $P_{i}$ of the receiver $R_{i}$ can be computed as:

$$
P_{i}=1-\left(1-p_{i}\right)^{N}
$$

where $N$ denotes the packet size in bits. We now proceed with the bandwidth efficiency of ARQ technique for broadcast.

1) ARQ Broadcast: Let $X_{1}$ and $X_{2}$ be the random variables denoting the number of attempts to successfully deliver a packet to $R_{1}$ and $R_{2}$, respectively. Thus, the number of transmissions needed to deliver a packet successfully to all receivers is a random variable $Y=\max _{i \in\{1,2\}}\left\{X_{i}\right\}$. From Equation (1), the probability of $j$ or fewer required transmissions is

$$
\begin{aligned}
P[Y \leq j] & =P\left[\max _{i \in\{1,2\}}\left\{X_{i}\right\} \leq j\right] \\
& =\prod_{i=1}^{2} P\left[X_{i} \leq j\right]=\prod_{i=1}^{2}\left(1-P_{i}^{j}\right) .
\end{aligned}
$$

Therefore,

$$
P[Y=j]=\prod_{i=1}^{2}\left(1-P_{i}^{j}\right)-\prod_{i=1}^{2}\left(1-P_{i}^{j-1}\right) .
$$

The expected number of transmissions to deliver a successful packet to all the receivers can then be computed as:

$$
\begin{aligned}
E[Y] & =\sum_{j=1}^{\infty} j\left(\prod_{i=1}^{2}\left(1-P_{i}^{j}\right)-\prod_{i=1}^{2}\left(1-P_{i}^{j-1}\right)\right) \\
& =\sum_{j=1}^{\infty} j\left(P_{1}^{j-1}-P_{1}^{j}\right)+\sum_{j=1}^{\infty} j\left(P_{2}^{j-1}-P_{2}^{j}\right) \\
& +\sum_{j=1}^{\infty} j\left(P_{1}^{j} P_{2}^{j}-P_{1}^{j-1} P_{2}^{j-1}\right) \\
& =\frac{1}{1-P_{1}}+\frac{1}{1-P_{2}}-\frac{1}{1-P_{1} P_{2}}
\end{aligned}
$$

Or equivalently, the broadcast bandwidth efficiency $\eta_{B A}$ of the ARQ technique is

$$
\eta_{B A}=\frac{N-r}{N\left(\frac{1}{1-P_{1}}+\frac{1}{1-P_{2}}-\frac{1}{1-P_{1} P_{2}}\right)}
$$

2) $A R Q$ Unicast: For unicast scenario, each receiver wants to receive $M$ distinct packets. so, the unicast bandwidth efficiency $\eta_{U A}$ can be easily derived as:

$$
\eta_{U A}=\frac{2(N-r)}{N\left(\frac{1}{1-P_{1}}+\frac{1}{1-P_{2}}\right)}=\frac{2(N-r)\left(1-P_{1}\right)\left(1-P_{2}\right)}{N\left(2-P_{1}-P_{2}\right)}
$$

\section{B. HARQ Technique}

In this section, we derive the bandwidth efficiency for a simple Type-I HARQ technique [20] when using Reed Solomon code $R S(n, k)$ for error correction and $r$ CRC bits for error detection. We assume that the symbol length is $m$ bits and each packet consists of $X$ code blocks. Upon receiving a packet, the receiver first performs the error correction using $R S(n, k)$ then error checking (detection) using CRC bits. At the receiver, we omit the use of Chase Combining (CC) [20] in decoding for ease of analysis. For the broadcast scenario, we assume that all the packets are of same size and have the same FEC protection levels. For the unicast scenario, the packet size is also assumed fixed, while the FEC protection levels may vary for different receivers. We now begin with the broadcast scenario.

1) HARQ Broadcast: Given that the symbol length is $m$ bits, the Symbol Error Probability (SEP), i.e., the probability of one or more bits are corrupted within a symbol for a receiver $R_{i}$ is:

$$
S E P_{i}=1-\left(1-p_{i}\right)^{m}
$$

Therefore, the irrecoverable packet error probability $P_{i}^{f}$ for receiver $R_{i}$ after using $R S(n, k)$, is:

$$
P_{i}^{f}=1-\left(\sum_{j=0}^{t}\left(1-S E P_{i}\right)^{n-j} S E P_{i}^{j}\right)^{X}
$$

where $t=\left\lfloor\frac{n-k}{2}\right\rfloor$.

Since $L=k . m . X-r$ and $N=n . m . X$ are the number of data bits and total bits in a packet, the bandwidth efficiency $\eta_{F}$ for HARQ technique can be computed similar to the ARQ techniques as:

$$
\eta_{F}=\frac{1}{\left(\frac{1}{1-P_{1}^{f}}+\frac{1}{1-P_{2}^{f}}-\frac{1}{1-P_{1}^{f} \cdot P_{2}^{f}}\right)} \frac{L}{N}
$$

2) HARQ Unicast: For the unicast scenario, the protection levels may vary for different receivers. Let $R S\left(n, k_{1}\right)$ and $R S\left(n, k_{2}\right)$ be the $R S$ codes used to protect packets destined for receivers $R_{1}$ and $R_{2}$, respectively. Hence, the maximum number of error symbols at a receiver $R_{i}$ that can be corrected is $t_{i}=\left\lfloor\frac{n-k_{i}}{2}\right\rfloor$. Then, the probability of an irrecoverable packet loss $P_{i}^{f}$ at $R_{i}$ is given by,

$$
P_{i}^{f}=1-\left(\sum_{j=0}^{t_{i}}\left(1-S E P_{i}\right)^{n-j} S E P_{i}^{j}\right)^{X}
$$

Let $N=n . m . X$ and $L_{i}=k_{i} \cdot m . X-r$ denote the total number of bits and the number of data bits in a packet for receiver $R_{i}$, 


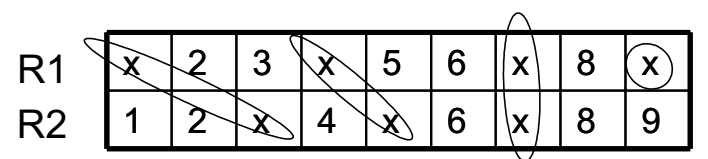

Fig. 2. Combined packets for time-based retransmission: $a_{1} \oplus a_{3}, a_{4} \oplus a_{5}$, $a_{7}, a_{9} ; M=9$

then the unicast bandwidth efficiency for two receivers can be computed as:

$$
\eta_{U F}=\frac{\left(L_{1}+L_{2}\right)}{\frac{N}{1-P_{1}^{f}}+\frac{N}{1-P_{2}^{f}}}=\frac{\left(L_{1}+L_{2}\right)\left(1-P_{1}^{f}\right)\left(1-P_{2}^{f}\right)}{N\left(1-P_{2}^{f}\right)+N\left(1-P_{1}^{f}\right)}
$$

\section{Network Coding Technique}

In [6], we proposed a NC scheme as follows. The receiver's protocol is similar to that of the receiver in the ARQ scheme in which it sends the NAK immediately if it does not receive a packet correctly. However, the source does not retransmit the lost packet immediately when it receives a NAK. Instead, the source maintains a list of lost packets and the corresponding receivers for which their packets are lost. The retransmission phase starts at a fixed interval of time in terms of the number of time slots. During the retransmission phase, the source forms a new packet by XORing a maximum set of the lost packets from different receivers before retransmitting this combined packet to all the receivers. Even though a receiver successfully receives the combined packets, it must be able to recover the lost packets, and it does so by XORing this combined packets with appropriate set of previously successful packets. The information on choosing this appropriate set of packets is included in the packets sent by the source. For example, Fig. 2 shows a pattern of lost packets (denoted by the crosses) for two receivers $R_{1}$ and $R_{2}$. The combined packets are $a_{1} \oplus a_{3}$, $a_{4} \oplus a_{5}, a_{7}, a_{9}$, where $a_{i}$ denotes the $i^{\text {th }}$ packet. Receiver $R_{1}$ recovers packet $a_{1}$ as $a_{3} \oplus\left(a_{1} \oplus a_{3}\right)$. Similarly, receiver $R_{2}$ recovers packet $a_{3}$ as $a_{1} \oplus\left(a_{1} \oplus a_{3}\right)$. When the same packet loss occurs at both receivers $R_{1}$ and $R_{2}$, the encoding process is not needed and the source just has to retransmit that packet alone. Note that the source has to include some bits to indicate to a receiver which set of packets it should use for XORing. In [6], we have shown that the bandwidth efficiency $\eta_{B N}$ for a broadcast session is

$$
\eta_{B N}=\frac{\left(1-\max \left\{P_{1}, P_{2}\right\}\right)(N-r)}{N}
$$

1) NC Unicast: We now extend the network coding technique to unicast setting. Assume that $R_{1}$ wants to receive packet $a_{1}$ while $R_{2}$ wants to receive packet $a_{2}$. Clearly, if $R_{1}$ is willing to cache packet $a_{2}$ intended for $R_{2}$, and $R_{2}$ is willing to cache packet $a_{1}$ intended for $R_{1}$, then the two unicast sessions are now equivalent to a single broadcast session. Similarly, when there are $R$ receivers that want to receive different packets, a receiver may want to cache everyone else's data in order to use network coding for higher bandwidth efficiency. However, unlike the broadcast scenario with two receivers in which, a combined packet can be an XORed packet of any lost packets, in the unicast scenario, the combined packet must be a XOR combination of an even and an odd packet in order to be advantageous. This is because each receiver is only interested in receiving its own packets. For example, consider the loss patterns depicted in Fig. 2 where $R_{1}$ and
$R_{2}$ want to receive odd and even packets respectively. In this case, it is not advantageous to XOR packets $a_{1}$ and $a_{3}$ even though one successful transmission of this combined packet may allow $R_{1}$ to recover packet $a_{1}$ and $R_{2}$ to recover $a_{3}$. This is because $R_{2}$ does not want $a_{3}$, and $a_{3}$ will never be used in subsequent packet combining since $R_{1}$ already had packet $a_{3}$. Thus, the sender may as well send packet $a_{1}$ to avoid unnecessary coding. Using this unicast scheme, we have the following proposition:

Proposition 4.1: The bandwidth efficiency when using network coding technique for two receivers with packet loss rates $P_{1}$ and $P_{2}$ is:

$$
\eta_{U N}=\frac{2\left(1-P_{2}\right)(N-r)}{N\left(2-P_{2}\right)}
$$

where $P_{1} \leq P_{2}$ and $M$, the number of packets destined for each receiver is sufficiently large.

Proof:

Without loss of generality, assume that the receivers $R_{1}$ and $R_{2}$ want to receive the $M$ odd and $M$ even packets, respectively. The bandwidth gain of the network coding technique depends on how many pairs of lost packets among the two receivers that one can find in order to generate the combined packets. When the number of packets $M$ to be sent is sufficiently large, the probability that the number of lost packets at $R_{1}$ is smaller than or equal to that of $R_{2}$, is close to 1 since $P_{1} \leq P_{2}$ by assumption. Furthermore, the average numbers of lost packets for $R_{1}$ and $R_{2}$ are $M P_{1}$ and $M P_{2}$, respectively. The retransmitted packets can be classified into two types: the combined and non-combined packets. As discussed previously, the sender only combines odd and even lost packets. This implies that on average the number of packets one can pair up is $\min \left(M P_{1}, M P_{2}\right)=M P_{1}$. As a result, there are $M P_{2}-M P_{1}$ lost packets from $R_{2}$ that need to be retransmitted as non-combined packets. Hence, the total number of transmissions needed to deliver $M$ packets to each receiver successfully is

$$
T=2 M+M P_{1} \cdot E\left[X_{1}\right]+\left(M P_{2}-M P_{1}\right) \cdot E\left[X_{2}\right]
$$

where $X_{1}$ and $X_{2}$ are the random variables denoting the numbers of attempts before a successful transmission for the combined packets and non-combined packets, respectively. $X_{2}$ follows the geometric distribution, $E\left[X_{2}\right]=\frac{1}{1-P_{2}}$. Now, one can think of $E\left[X_{1}\right]$ as the expected number of transmissions per successful transmission in the NC broadcast scheme in which, the sender must transmit successfully a combined packet to both receivers. Therefore, from Equation (11), we have

$$
E\left[X_{1}\right]=\frac{1}{1-\max \left\{P_{1}, P_{2}\right\}}=\frac{1}{1-P_{2}}
$$

Substituting $E\left[X_{1}\right]$ and $E\left[X_{2}\right]$ into (13) and dividing it by $M$ we have the expected number of transmissions to successfully deliver two packets to $R_{1}$ and $R_{2}$ as:

$$
\eta_{U N}^{1}=2+\frac{P_{2}}{1-P_{2}}
$$

Consequently, the bandwidth efficiency for NC unicast coding is

$$
\eta_{U N}=\frac{2(N-r)}{N\left(2+\frac{P_{2}}{1-P_{2}}\right)}=\frac{2(N-r)\left(1-P_{2}\right)}{N\left(2-P_{2}\right)}
$$


We can generalize the above result to $R$ receivers.

Theorem 4.1: The network bandwidth efficiency for $R$ receivers and sufficiently large $M$ is:

$$
\eta_{U N}=\frac{(N-r) R\left(1-\max _{i \in\{1, \ldots, R\}}\left\{P_{i}\right\}\right)}{N\left(R-(R-1) \max _{i \in\{1, . ., R\}}\left\{P_{i}\right\}\right)}
$$

Proof: Due to page limitation, the proof can be found in [19].

\section{Joint NC and FEC (NC-HARQ) Technique}

NC-HARQ technique employs for NC and FEC for reliable transmission. However, instead of using ARQ when a packet is lost, it uses the NC technique described in Section IV$\mathrm{C}$ for retransmission. Also, we assume that each receiver uses the same packet size and protection level in case of wireless broadcast scenario. When the sender needs to send out a combined packet, it first performs XOR on the data before adding the FEC. Conversely, upon receiving a combined packet, the receiver first decodes the data before performing XOR to recover the lost packet ${ }^{1}$. We now begin with an analysis of the NC-HARQ broadcast scenario.

1) NC-HARQ Broadcast: In [6], for broadcast scenarios, we have shown that $\mathrm{NC}$ technique is always better than ARQ technique in terms of bandwidth efficiency, regardless of network conditions. Thus, it is straightforward to see that NC-HARQ technique should always be better than HARQ technique. Intuitively, this is because the HARQ technique essentially transforms an error-prone channel into a more reliable channel by adding FEC, then using ARQ technique to retransmit the remaining packet losses. The NC-HARQ technique also uses FEC to improve the channel quality while employing a better retransmission technique, i.e., NC, thus its performance should be better than the HARQ scheme. We have the following corollary:

Corollary 4.1: The bandwidth efficiency of using NC$H A R Q$ broadcast scheme with $R$ receivers and irrecoverable packet loss rates $P_{1}^{f}, P_{2}^{f}, \ldots P_{R}^{f}$ is:

$$
\eta_{B N F}=\left(1-\max _{i \in\{1 \ldots R\}} P_{i}^{f}\right) \frac{L}{N}
$$

where $N=n . m . X+r$ and $L=k . m . X$ are the total bits and data bits in a packet, respectively.

2) NC-HARQ Unicast: For unicast scenario, we assume that each receiver uses the same packet size $N$, but the protection levels may vary for different receivers. Using a similar argument as the one in Section IV-C.1, we have the following corollary on the bandwidth efficiency for NC-HARQ unicast.

Corollary 4.2: The bandwidth efficiency of using NC$H A R Q$ unicast scheme with $R$ receivers and irrecoverable packet loss rates $P_{1}^{f}, P_{2}^{f}, \ldots P_{R}^{f}$ is:

$$
\eta_{U N F}=\frac{\left(1-\max _{i \in\{1, . ., R\}}\left\{P_{i}^{f}\right\}\right) \sum_{i=1}^{R} L_{i}}{\left(R-(R-1) \max _{i \in\{1, . ., R\}}\left\{P_{i}^{f}\right\}\right) N}
$$

where $L_{i}=k_{i} . m . X$ denotes the number of data bits in a packet of receiver $R_{i}$.

\footnotetext{
${ }^{1}$ If addition in finite field is used instead of XOR, one can also reverse the order of addition and channel code since given two packets $a_{1}, a_{2}$, and a generator $G, G\left(a_{1}+a_{2}\right)=G a_{1}+G a_{2}$.
}

Note that the irrecoverable packet loss rate $P_{i}^{f}$ can be easily computed from the bit error rate $p_{i}$ and the amount of protection, as expressed in Eq. (9).

\section{Heuristic Transmission Algorithm}

Up until now, we have presented the theoretical results on the bandwidth efficiencies for different schemes. Theoretically, we can show that the followings are true: (1) The NC-HARQ technique is always better than the HARQ technique in terms of the bandwidth efficiency under identical channel conditions and the same amount of redundancy; (2) The NC technique is always better than the ARQ technique under identical channel conditions. However, without the channel characteristics, one cannot determine whether NC-HARQ or NC techniques is better. On the other hand, NC is a special case of NCHARQ where redundant information is not added. Thus, the optimal technique is the NC-HARQ technique with the right amount of redundancy for given channel characteristics. Based on this, we propose the following heuristic scheme which dynamically uses the appropriate amount of redundancy for NC-HARQ technique, depending on channel conditions. In order to be fast, our algorithm relies on a look-up table which stores the tuple of bit error rates and the corresponding optimal redundancies for each receivers. The bit error rates are quantized into a certain step size, and the corresponding optimal redundancies are computed off-line using the theoretical results in Section IV-D.

Our algorithm estimates the bit error rates for each receiver periodically and uses these information to index into the lookup table to obtain the corresponding optimal redundancies. Next, the algorithm applies NC-HARQ techniques appropriately for either broadcast or unicast scenarios. One drawback of the current algorithm is that the table look-up can be exponentially large with the number of receivers and the quantization bins for the bit error rates. A solution would be to compute the optimal redundancies on the fly, thus eliminating the need for storage.

\section{Vi. Simulations AND Discussions}

In this section, we first present the simulation results on the bandwidth efficiencies of different techniques. To simulate the transmissions in a WLAN, we would like to set the packet size approximately around 1500 bytes. However, when using such a large packet size under a large BER, e.g. on the order of $10^{-3}$, the bandwidth efficiencies of the ARQ and NC schemes are much worse than those of the HARQ and NC-HARQ schemes. To be fair, we use smaller packet size, i.e., 222 bytes for ARQ and NC schemes, and also incorporate a very light protection using $R S(127,123)$. For HARQ and NC-HARQ schemes, the packet size is set at 1559 bytes (WLAN packet size) and data is encoded with $R S(127,114)$. In addition, for unicast, we allow each receiver in different schemes to have different levels of error protection. In particular, HARQ and NC-HARQ schemes employ $R S(127,114)$ and $R S(127,116)$, while $N C$ and $A R Q$ schemes employ a slight protection $R S(127,123)$ and $R S(127,125)$ for two receivers. We use CRC-32 for error detection in all the simulations. Fig. 3(a) and Fig. 3(b) show the simulation and theoretical bandwidth efficiency as a function of bit error rate for broadcast and unicast sessions with one sender and two receivers. The bit 


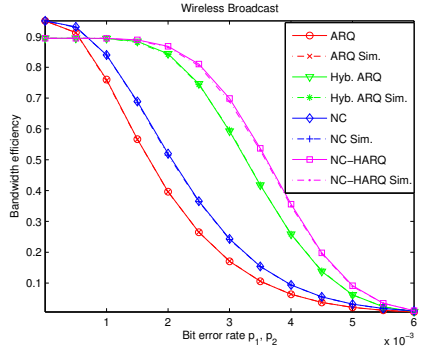

(a)

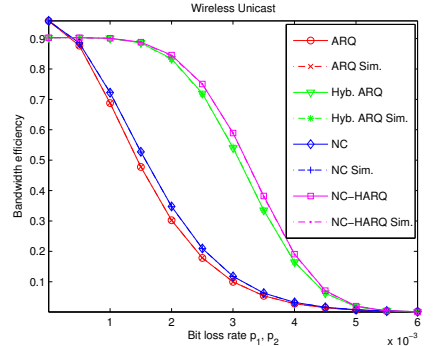

(b)
Fig. 3. Bandwidth efficiency versus BER for theory and simulation (a) broadcast and $(b)$ unicast.

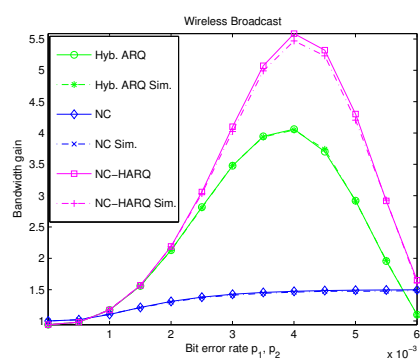

(a)

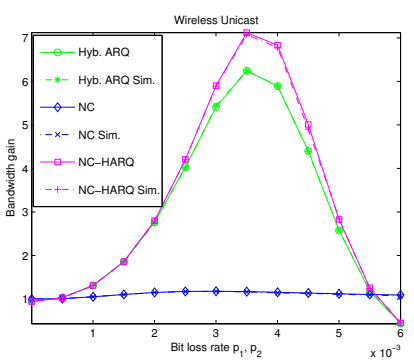

(b)
Fig. 4. Bandwidth gain over $A R Q$ technique versus BER for theory and simulation for (a) broadcast and (b) unicast.

error rates of two receivers vary from $10^{-6}$ to $6 \times 10^{-3}$. As seen, the simulation results verify our theoretical derivations. Furthermore, as predicted, the NC-HARQ scheme always outperforms the HARQ scheme and the NC scheme always outperforms the ARQ scheme. In small BER regions, the $\mathrm{NC}$ scheme performs the best which is intuitively plausible since redundancy introduced by the NC-HARQ scheme would just increase the bandwidth overhead unnecessarily. Similarly, Fig. 3(b) shows the bandwidth efficiency versus BER for the wireless unicast scenario. Figures 4(a) and 4(b) show the bandwidth gains of FEC, NC, NC-HARQ schemes over the ARQ scheme for broadcast and unicast scenarios. The bandwidth gain of scheme $A$ over $B$ is defined as the ratio of the bandwidth efficiency of $A$ over that of $B$. As seen, for some BER region, the proposed NC-HARQ technique can be more than five times efficient than ARQ technique. We now compare the performance of the proposed dynamic NC-HARQ algorithm against other schemes. The channel condition is simulated to change with time. In particular, $p_{1}$ varies from $10^{-6}$ to $4 \times 10^{-3}$ with a step size of $2 \times 10^{-4}$ while $p_{2}$ randomly changes in $\left[5 \times 10^{-7}, 2.5 \times 10^{-3}\right]$. All the parameters except packet size are identical to the previous simulations for all the non-adaptive schemes. The packet size are set to 1337 bytes for HARQ and NC-HARQ schemes and 337 bytes for NC and ARQ schemes. Fig. 5(a) and (b) show the bandwidth gains over ARQ technique as a function of $p_{1}$ for different schemes in the broadcast and unicast scenarios, respectively. As seen, the dynamic NC-HARQ algorithm has the best performance as it can adapt the amount of redundancy appropriately.

\section{CONCLUSIONS}

We have proposed a joint network-channel coding technique to increase bandwidth efficiency of single-hop wireless networks for both broadcast and unicast scenarios. The theoretical

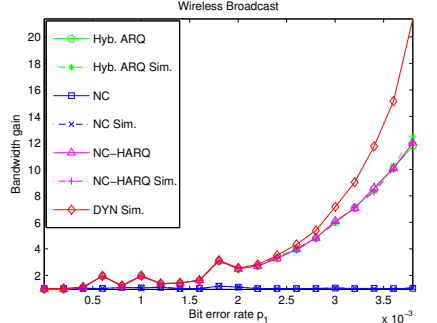

(a)

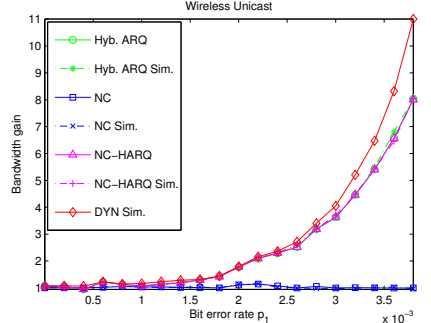

(b)
Fig. 5. Bandwidth gain of different techniques under changing network conditions for (a) broadcast and (b) unicast.

and simulation results showed that our proposed technique can efficiently utilize high bandwidth over those of traditional techniques for a typical range of channel conditions.

\section{REFERENCES}

[1] J. Clark Jr. and J. Cain, Error-Correction Coding for Digital Communications, New York: Plenum, 1982.

2] Y. Wu, P. A. Chou, and S.-Y. Kung, "Information exchange in wireless networks with network coding and physical-layer broadcast," in Technical Report MSR-TR-2004-78, Microsoft Research, Aug. 2004.

[3] Sachin Katti, Hariharan Rahul, Wenjun Hu, Dina Katabi, and Muriel Medard Jon Crowcroft, "Xors in the air: Practical wireless network coding," in ACM SIGCOMM.

[4] Y. Wu, P. A. Chou, and S.-Y. Kung, "Minimum-energy multicast in mobile ad hoc networks using network coding," in IEEE Information Theory Workshop, Oct. 2004.

[5] S. Deb, M. Effros, T. Ho, D. R. Karger, R. Koetter, D. S. Lun, M. Medard, and N. Ratnakar, "Network coding for wireless applications: A brief tutorial," in in IWWAN, 2005.

[6] Dong Nguyen, Thinh Nguyen, and Bella Bose, "Wireless broacast using neotwork coding," in NetCod Workshop, january 2007.

[7] S Katti, D Katabi, W Hu, H Rahul, and M. Medard, "The importance of being opportunistic: Practical network coding for wireless environments," in Proc. 43rd Annual Allerton Conference on Communication, 2005.

[8] C. Fragouli, J. Le Boudec, and J. Widmer, "Network coding: An instant primer," in Technical Report, TR2005010, EPFL, 2005.

[9] Atilla Eryilmaz, Asuman Ozdaglar, and Muriel Medard, "On delay performance gains from network coding," in 40th Annual Conference on Information Sciences and Systems, 2006.

[10] T. Ho, M. Medard, J. Shi, M. Effros, and D. R. Karger, "On randomized network coding," in in Proc. 41st Annual Allerton Conference on Communication, Control, and Computing, October 2003.

[11] T. Ho, M. Medard, D. R. Karger, M. Effros, J. Shi, and B. Leong, "A random linear network coding approach to multicast," IEEE Trans. Inform. Theory, 2004.

[12] M. Ghaderi, D. Towsley, and Jim Kurose, "Reliability benefit of network coding," in Tech. Report 07-08, Computer Science Department, University of Massachusetts Amherst, February 2007.

[13] B. Li Z. Li, "On increasing end-to-end thoughput in wireless ad hoc networks," in Conference on Quality of Service in Heterogeneous Wired/Wireless Networks (QShine), 2005.

[14] Z. Li and B. Li, "Network coding: the case for multiple unicast sessions," in Allerton Conference on Communications, 2004.

[15] D. Lun, M. Medard, R. Koetter, and M. Effros, "On coding for reliable communication over packet networks," 2005.

[16] A. Shiozaki, "Adaptive type-ii hybrid broadcast arq system," IEEE Transactions on Communications, vol. 44, pp. 420-422, April 1996.

[17] S.R. Chandran and S. Lin, "Selective-repeat-arq schemes for broadcast links," IEEE Transactions on Communications, vol. 40, pp. 12-19, January 1992.

[18] S. Kallel and D. Haccoun, "Generalized type ii hybrid arq scheme using punctured convolutional codes," IEEE Transactions on Communications, vol. 38, pp. 1938 - 1946, november 1990.

[19] T. Tran, "A joint network and channel coding technique for wireless networks," in Technical Report: OSU-TR-2007-06, Oregon State University, June 2007.

[20] Stephen Wicker, Error Control Systems for Digital Communication and Storage, Prentice-Hall, 1995. 